Original Article

\title{
Immunohistochemical expression of keratan sulfate: a possible diagnostic marker for carcinomas of the female genital tract
}

Running title: A novel diagnostic marker, KS

Tsutomu Miyamoto, M.D., Ph.D.* ${ }^{1}$, Keiko Ishii, M.D., Ph. D. ${ }^{2}$, Ryouichi Asaka, M.D. ${ }^{1}$, Akihisa Suzuki, M.D. ${ }^{1}$, Akiko Takatsu, M.D. ${ }^{1}$, Hiroyasu Kashima, M.D., Ph.D. ${ }^{1}$ and Tanri Shiozawa, M.D., Ph.D. ${ }^{1}$

1: Department of Obstetrics and Gynecology, Shinshu University School of Medicine, 3-1-1 Asahi, Matsumoto 390-8621, Japan

2: Division of Diagnostic Pathology, Okaya City Hospital, 4-11-33 Honmachi, Okaya 394-8512, Japan

* Address correspondence to: T. Miyamoto, Department of Obstetrics and Gynecology, Shinshu University School of Medicine, 3-1-1 Asahi, Matsumoto 390-8621, Japan

Key words: Keratan sulfate, diagnostic marker, female genital tract, carcinoma, endometriosis

Word count: 1,969 words 


\section{ABSTRACT}

Aims: We previously reported the expression of keratan sulfate (KS), a glycosaminoglycan, in the epithelium of normal and neoplastic endometria. Here, to evaluate its potential use as a diagnostic marker, the expression of KS in other human epithelial tissues was investigated.

Methods: The expression was examined immunohistochemically using 102 samples of normal epithelia and 110 samples of carcinomas from the female genital tract (FGT; cervix, endometrium, ovary, fallopian tube), digestive organs (DO; gastrointestinal tract, pancreas, liver), urinary tract (UT), lung, mammary gland, thyroid and mesothelium.

Results: In normal tissues, KS was consistently detected in the FGT and ectopic endometrium (25/26), but not found in the DO (1/42) and UT (0/6), and only partial detected in the lung (7/10), mammary gland (3/9) and thyroid (4/4). In malignant tissues, KS was consistently observed in carcinomas of the endometrium, ovary and fallopian tube (29/32), and partially detected in carcinomas of the lung, mammary gland, thyroid, pancreas and mesothelium, but abcent in carcinomas of the gastrointestinal tract (0/17), liver (0/5) and UT (0/11). Among carcinomas of the FGT, DO and UT, KS-positivity suggested the possibility of FGT carcinomas with 79.5\% (31/39) sensitivity and 92.9\% (39/42) specificity.

Conclusions: KS is a potentially useful marker for the supportive diagnosis of the primary site of metastatic carcinomas or unknown primary carcinomas, especially in the abdominal cavity. 


\section{INTRODUCTION}

Many proteins and lipids are linked to sugar chains to form glycoconjugates including glycoprotein, glycolipid and proteoglycan. Glycoconjugates constitute a large number of cell surface markers in various stages of differentiation with various functions. For instance, blood type antigens and many tumor markers such as CA125 (carbohydrate antigen 125) and CA19-9 are included among cell surface glycoconjugates.[1,2]

Glycosaminoglycans (GAGs) are long unbranched polysaccharide chains consisting of a repeating disaccharide unit. Typically, GAGs are covalently linked to a core protein to form proteoglycans (PGs). PGs hold many water molecules, which function to resist physical pressure. Therefore, GAGs and PGs are important components of connective tissues like cartilage.[3] Keratan sulfate (KS) is a GAG containing a disaccharide unit composed of $\mathrm{N}$-acetylglucosamine and galactose, which are often sulfated. KS is also an important component of the matrix of cartilage, carnea and brain.[3]

We previously reported that KS was expressed in the epithelium of eutopic and ectopic endometria and endometrial carcinomas, and suggested the expression to be a potential marker for ectopic endometrium including colonic endometriosis.[4] However, there are few reports of KS expression in the epithelium of normal tissues, or in other neoplastic tissues. Although several histopathological markers such as CA125, cytokeratin (CK) 7 and CK20 are currently used for the supportive diagnosis of tumors, identification of the primary site of a metastatic carcinoma or an unknown primary carcinoma is often difficult. This background indicates KS to be a novel marker for distinguishing tumors originating from the female genital tract (FGT) from carcinomas of other primary sites. Therefore, in the present study, the immunohistochemical 
expression of KS was extensively investigated in various normal and malignant epithelial tissues to clarify the utility of KS as a diagnostic tool.

\section{MATERIALS AND METHODS}

\section{Samples}

Formalin-fixed and paraffin-embedded tissue specimens obtained by surgical resection or biopsy were selected from the pathology files of Shinshu University Hospital, and used for immunohistochemistry. For the search for KS expression in non-neoplastic epithelium, we selected cases as follows; 27 FGT (11 endometria, 3 cervixes, 3 fallopian tubes, 3 ovaries, 7 ectopic endometria), 46 digestive organs (DO; 6 esophagi, 6 stomachs, 15 small/large intestines, 6 pancreases, 7 livers, 3 gall bladders, 3 bile ducts), 6 urinary tracts (UT; 2 kidneys, 4 urinary bladders), 10 lungs, 9 mammary glands and 4 thyroids. In addition, to examine the expression of KS in carcinomas, we selected cases as follows; 17 endometrial carcinomas (12 endometrioid, 2 serous, 2 clear cell and 1 anaplastic), 7 cervical carcinomas (5 adenocarcinomas, 2 squamous cell carcinomas), 10 ovarian carcinomas (2 endometrioid, 4 serous, 2 clear cell and 2 mucinous), 5 tubal and primary peritoneal carcinomas, 17 carcinomas of gastrointestinal tract (GIT; 5 esophagus, 5 stomachs, 7 small/large intestine), 9 pancreatic carcinomas, 5 hepatic carcinomas, 11 carcinomas of UT (5 kidneys, 6 urinary bladders) and 29 other cancers (9 lung, 10 mammary gland, 5 thyroid and 5 malignant mesotheliomas). Each tissue sample was used with the approval of the Ethics Committee of Shinshu University.

\section{Immunohistochemistry}

Indirect immunohistochemical staining was performed on paraffin sections using a mouse monoclonal anti-KS antibody, 5D4 (Seikagaku Biobusiness, Tokyo, Japan) (1: 
2000), as a primary antibody and a horseradish peroxidase-conjugated goat anti-mouse IgG antibody (Dako, Glostrup, Denmark) (1: 50) as a secondary antibody. After antigen retrieval by microwave pretreatment, the sections were incubated with the primary antibody and secondary antibody each for 1 hour at room temperature. Then, the immunocomplex bound to KS was visualized by diaminobenzidine. Weak counterstaining was performed with hematoxylin. The same sections were stained with hematoxylin and eosin (HE) for reference.

\section{Evaluation of immunoreactivity}

Immunoreactivity was evaluated according to the percentage of positive cells semiquantitatively by two independent reviewers (T.M. and T.S.). No staining in the target cells was defined as negative (-), staining in less than $5 \%$ of the cells was defined as weakly positive $(+)$, and staining in more than $5 \%$ of the cells was defined as positive $(2+)$.

\section{RESULTS}

\section{The expression of KS in the normal FGT and other epithelial tissues}

Immunoreactivity for KS was observed at the cell surface and in the cytoplasm. The expression of KS in normal epithelial tissues is summarized in Fig. 1. Staining for KS was often detected in normal (10/11, Figs. 2a, b) and ectopic (7/7, Figs. 2c, d) endometrial glands. KS was also expressed at other sites of the FGT including the cervix (endocervical glands; 3/3, squamous epithelium; 1/3), tubal epithelium (3/3, Figs. 2e, f), and ovarian inclusion cyst (2/2). In contrast, no immunoreactivity for KS was found in the DO (Fig. 1, Figs. 2g, h) and UT (Figs. 2i, j), except for one case of esophageal squamous epithelium. However, the expression of KS was observed in the 
bronchial epithelium (7/10, Figs. 2k, l), bronchial glands (3/3), mammary glands (3/9) and thyroid follicles (4/4).

\section{The expression of $\mathrm{KS}$ in carcinomas of the FGT}

The expression of KS in various carcinomas is summarized in Fig. 3. Although the intensity of the staining was heterogeneous even within the same carcinoma, the expression of KS was observed in most cases of endometrial carcinoma (16/17, Figs. 4a-d). The expression seemed to be decreased in poorly differentiated carcinomas compared with well differentiated carcinomas. One anaplastic carcinoma was negative for KS.

In contrast, the staining of KS in cervical carcinomas was observed in only 2 of 7 cases. In five adenocarcinomas, the expression of KS was detected only in one endometrioid-type adenocarcinoma.

The expression of KS was observed in epithelial ovarian carcinomas (9/10) of the endometrioid, clear cell, serous (Figure 4e, f) and mucinous type. KS was also found in tubal and primary peritoneal carcinomas (4/5, Figs 3, 4g and h).

\section{The expression of $\mathrm{KS}$ in carcinomas of other organs}

The expression of KS in carcinomas of other organs is also summarized in Fig. 3. As in normal tissues, no expression of KS was detected in carcinomas of the GIT (Figs. 5a-d), liver and UT (Figs. 5e, f). However, KS was detected in several lung (5/9, Figs. 5g, h), breast (4/10, Figs. 5i, j), thyroid (5/5) and pancreatic (3/9) carcinomas. Malignant mesotheliomas were also positive for KS (3/5, Figs. 5k, l).

In comparison with the FGT and other abdominal organs, the KS expression pattern of carcinomas of the upper FGT including endometrial, ovarian, tubal and peritoneal carcinomas was clearly different from that of the GIT, liver and UT (Fig. 3). 
Among carcinomas of the FGT, DO and UT, KS-positive staining indicated 79.5\% (31/39) sensitivity and 92.9\% (39/42) specificity for the supportive diagnosis of carcinomas of the FGT (Table 1). Because the positive rate for KS was lower in cervical carcinomas than other carcinomas of the FGT, sensitivity was calculated excluding cervical carcinomas and was 90.6\% (29/32). Also, specificity was calculated without including pancreatic carcinomas and was 100\% (33/33).

Table 1: The number of KS-positive or -negative cases among carcinomas of the FGT and DO and UT

\begin{tabular}{cccc} 
& FGT & DO and UT & total \\
\hline positive & 31 & 3 & 33 \\
negative & 8 & 39 & 38 \\
\hline total & 39 & 42 & 71
\end{tabular}

\section{DISCUSSION}

PGs containing KS are an important component of the matrix of cartilage and cornea.[3] However, the present study revealed that KS was broadly expressed in epithelial cells of the FGT. The expression of KS in endometrium had been reported previously by us and by Hoadley and colleagues.[4,5] The latter research group had additionally reported that the KS in endometrium was carried by the mucin1 (MUC1) glycoprotein and involved in regulating the embryo’s attachment.[6] This might explain why we detected KS expression throughout the FGT.

The present study revealed that the expression of KS in the normal epithelium 
of abdominal organs was observed only in the FGT, not in the DO and UT. In addition, it revealed that KS was expressed in most carcinomas of the FGT, and not in carcinomas of the GIT, liver and UT. These findings indicated KS to be a potentially useful marker for the supportive diagnosis of diseases of the FGT including endometriosis of the GIT or UT. To evaluate ectopic endometriosis, CD10 is already used for the detection of endometrial stromal cells.[7] However, CD10 expression might be induced in carcinomas of the GIT, the UT or other areas.[8-10] Therefore, in terms of specificity, KS may be a more useful marker than CD10.

In addition, our results suggested KS-positive staining to be useful for the differential diagnosis of carcinomas of the FGT (endometrium, ovary, tube) and those of the GIT, liver or UT. The data demonstrated the high sensitivity (90.6\%) and specificity (100\%) of KS-positive staining for the diagnosis of carcinomas of the FGT. It should be noted that only pancreatic carcinomas showed staining for KS (33.3\%) among adenocarcinomas of the abdominal organs other than the FGT.

Currently, several diagnostic markers such as cytokeratine (CK) 7 and CK20, caudal-type homeobox-2 (CDX-2), estrogen receptor (ER) and CA125 are used for distinguishing between carcinomas of the FGT and those of the gastrointestinal tract.[11-13]

CA125 is one of the most common markers of carcinomas of the FGT, and is thought to be useful for distinguishing these carcinomas from colonic carcinomas.[12] However, CA125 expression was reported to be observed in almost half of pancreatic carcinomas and cholangiocarcinomas, and about $10 \%$ of gastric carcinomas.[12]

CK7 and CK20 are considered useful for differentiating carcinomas of the FGT from colonic carcinomas; many endometrial or ovarian carcinomas show CK7-positive 
and CK20-negative staining, but colonic carcinomas are reportedly CK7-negative and CK20-positive.[11,12] However, these markers are thought unsuitable for distinguishing carcinomas of the FGT from gastric carcinomas or pancreatic carcinomas,[11,12] because these carcinomas are often CK7-positive and CK20-negative.

CDX2 is known as a highly sensitive and specific marker for colorectal carcinomas, and is not expressed in most carcinomas of the FGT.[12] However, CDX2 was reportedly expressed in approximately $20 \%$ of endometrioid type endometrial carcinomas and endometrioid/mucinous type ovarian carcinomas.[14]

ER is also known as a specific receptor in gynecological and breast carcinomas.[15,16] And ER is rarely expressed in the gastrointestinal tract and other DO.[12,16] However, the sensitivity of this marker may be relatively low. Our previous data revealed that the rate of ER-positive cases in endometrial carcinomas was 69.6\%.[15]

In comparison with other markers, the sensitivity and specificity of KS against carcinomas of the FGT are thought to be considerably high. Although the characteristics of the expression pattern for each marker are different, KS is considered useful for the diagnosis of the primary site of a metastatic carcinoma or an unknown primary carcinoma.

The present study revealed the utility of KS in cervical carcinomas to be limited in comparison with other carcinomas of the FGT, because the rate of KS-positive cervical carcinoma cases was relatively low. Among primary malignant tumors, our results suggested that KS has only limited utility as a diagnostic marker for distinguishing carcinomas of the FGT from lung, breast, pancreatic carcinomas and mesotheliomas. 
In conclusion, the present study examined the expression of KS in various normal and malignant epithelia, and demonstrated that KS was specifically expressed in FGT in the abdominal organs. Based on the results, we propose the use of KS as a new diagnostic marker. Our data strongly suggested that the immunostaining of KS was particularly useful for distinguishing carcinomas of the FGT (endometrium, ovary and tube) from carcinomas of the GIT, liver and UT, and for the diagnosis of ectopic endometriosis.

\section{ACKNOWLEDGEMENTS}

The authors are grateful to Kazuya Murata, Masaomi Takahashi and Yukihiro Kobayashi (Department of Laboratory Medicine, Shinshu University Hospital) for their excellent technical assistance.

This work was supported in part by Grants-in-aid for Scientific Research from the Japan Society for the Promotion of Science (No. 22591852 and No. 22591851).

\section{DECLARATION OF INTEREST}

We have no conflict of financial interest related to this manuscript.

\section{TAKE-HOME MESSAGE}

- We propose a novel diagnostic marker "Keratan sulfate (KS)" for distinguishing tumors of the female genital tract from those of other primary sites.

- KS was commonly expressed in normal and neoplastic epithelia of the female genital tract, but not in the gastrointestinal tract, liver and urinary tract.

- In addition, the KS positivity was thought to be useful for the diagnosis of endometriosis at the intestine and/or urinary tract. 


\section{REFERENCES}

1. Yin BW, Lloyd KO. Molecular cloning of the CA125 ovarian cancer antigen: identification as a new mucin, MUC16. J Biol Chem 2001;276:27371-5.

2. Klug TL, Salzman S, Quinn A, et al. Tumorigenicity in athymic mice of the human colon carcinoma cell line SW1116 expressing the tumor-associated antigenic determinant CA 19-9. Cancer Res 1984;44:5212-8.

3. Esko JD, Kimata K, Lindahl U. Proteoglycans and Sulfated Glycosaminoglycans. In: Varki A, Cummings RD, Esko JD, eds. Essentials of Glycobiology, $2^{\text {nd }}$ ed. Cold Spring Harbor, NY: Cold Spring Harbor Laboratory Press 2009:229-48

4. Shiozawa T, Tsukahara Y, Nakayama J, et al. Immunohistochemical reactivity of antikeratan sulfate monoclonal antibody 5D4 to various conditions of human endometrial tissues and its application as a useful marker for identifying endometrial epithelia. Gynecol Obstet Invest 1991;32:239-42.

5. Hoadley ME, Seif MW, Aplin JD. Menstrual-cycle-dependent expression of keratan sulphate in human endometrium. Biochem J1990;266:757-63.

6. Aplin JD, Hey NA, Graham RA. Human endometrial MUC1 carries keratan sulfate: characteristic glycoforms in the luminal epithelium at receptivity. Glycobiology 1998;8:269-76.

7. Potlog-Nahari C, Feldman AL, Stratton P, et al. CD10 immunohistochemical staining enhances the histological detection of endometriosis. Fertil Steril 2004;82:86-92.

8. Chu P, Arber DA. Paraffin-section detection of CD10 in 505 nonhematopoietic neoplasms. Frequent expression in renal cell carcinoma and endometrial stromal sarcoma. Am J Clin Pathol 2000;113:374-82.

9. Bahadir B, Behzatoglu K, Bektas S, et al. CD10 expression in urothelial carcinoma of the bladder. Diagn Pathol 2009;4:38. 
10. Abdou AG. CD10 expression in tumour and stromal cells of bladder carcinoma: an association with bilharziasis. APMIS 2007;115:1206-18.

11. Tot T. Adenocarcinomas metastatic to the liver: the value of cytokeratins 20 and 7 in the search for unknown primary tumors. Cancer 1999;85:171-7.

12. Dennis JL, Hvidsten TR, Wit EC, et al. Markers of adenocarcinoma characteristic of the site of origin: development of a diagnostic algorithm. Cancer ReS 2005;11:3766-72.

13. Varadhachary GR, Abbruzzese JL, Lenzi R. Diagnostic strategies for unknown primary cancer. Cancer 2004;100:1776-85.

14. Moskaluk CA, Zhang H, Powell SM, et al. Cdx2 protein expression in normal and malignant human tissues: an immunohistochemical survey using tissue microarrays. Mod Pathol 2003;16:913-9.

15. Li SF, Shiozawa T, Nakayama K, et al. Stepwise abnormality of sex steroid hormone receptors, tumor suppressor gene products (p53 and $\mathrm{Rb}$ ), and cyclin $\mathrm{E}$ in uterine endometrioid carcinoma. Cancer 1996;77:321-9.

16. Lee BH, Hecht JL, Pinkus JL, et al. WT1, estrogen receptor, and progesterone receptor as markers for breast or ovarian primary sites in metastatic adenocarcinoma to body fluids. Am J Clin Pathol 2002;117:745-50. 
Figure 1

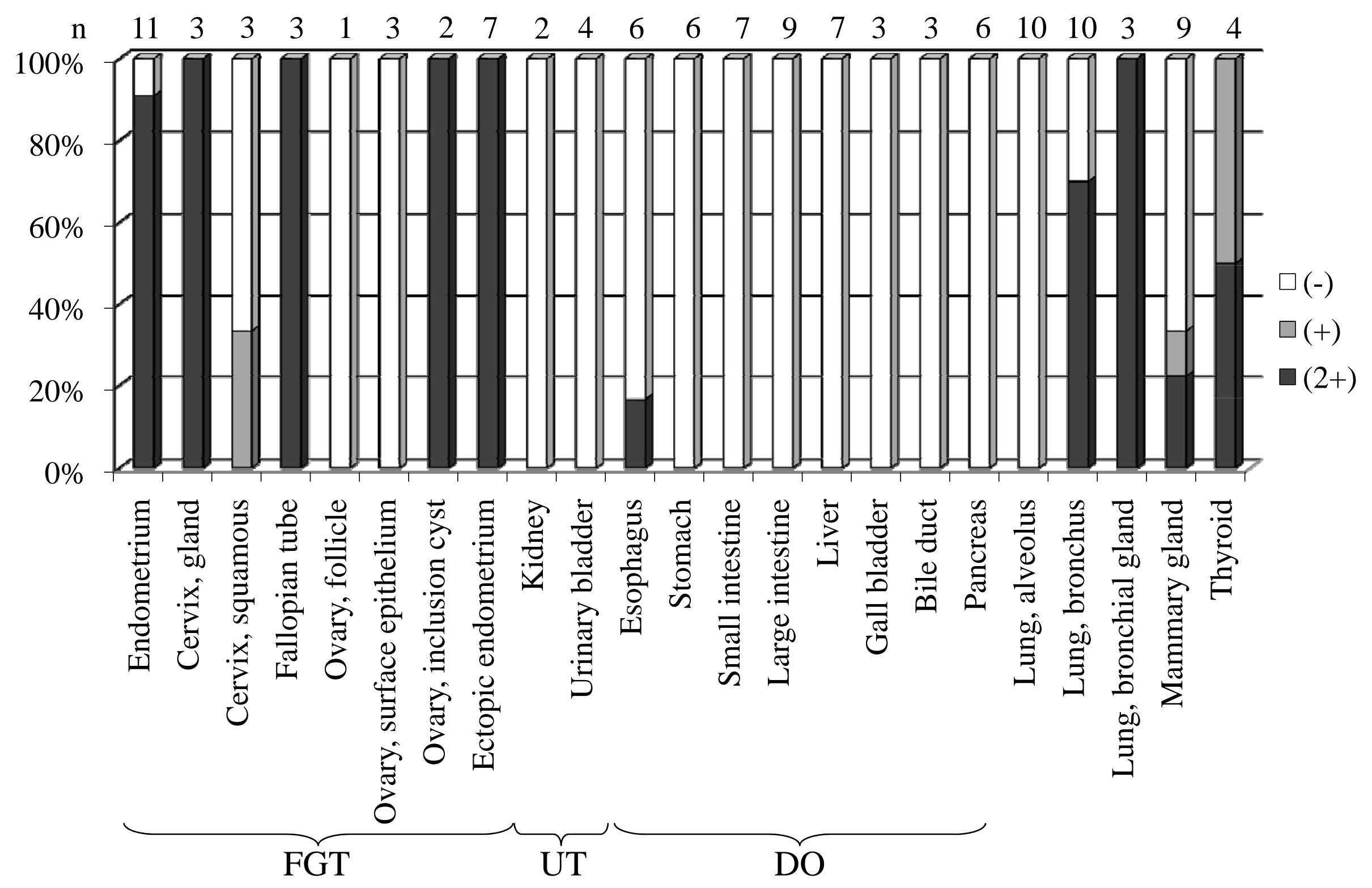


Figure 2
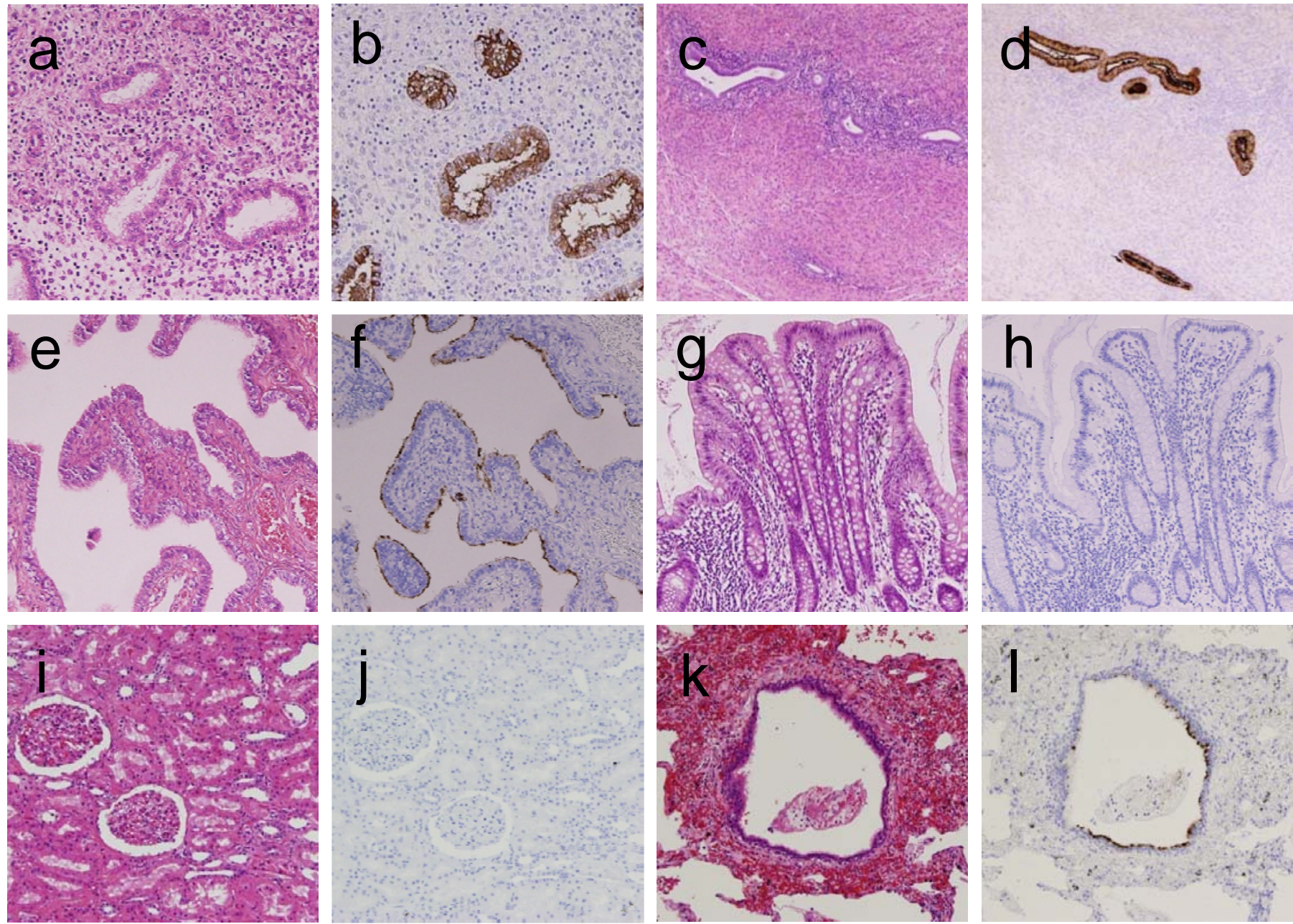


\section{Figure 3}

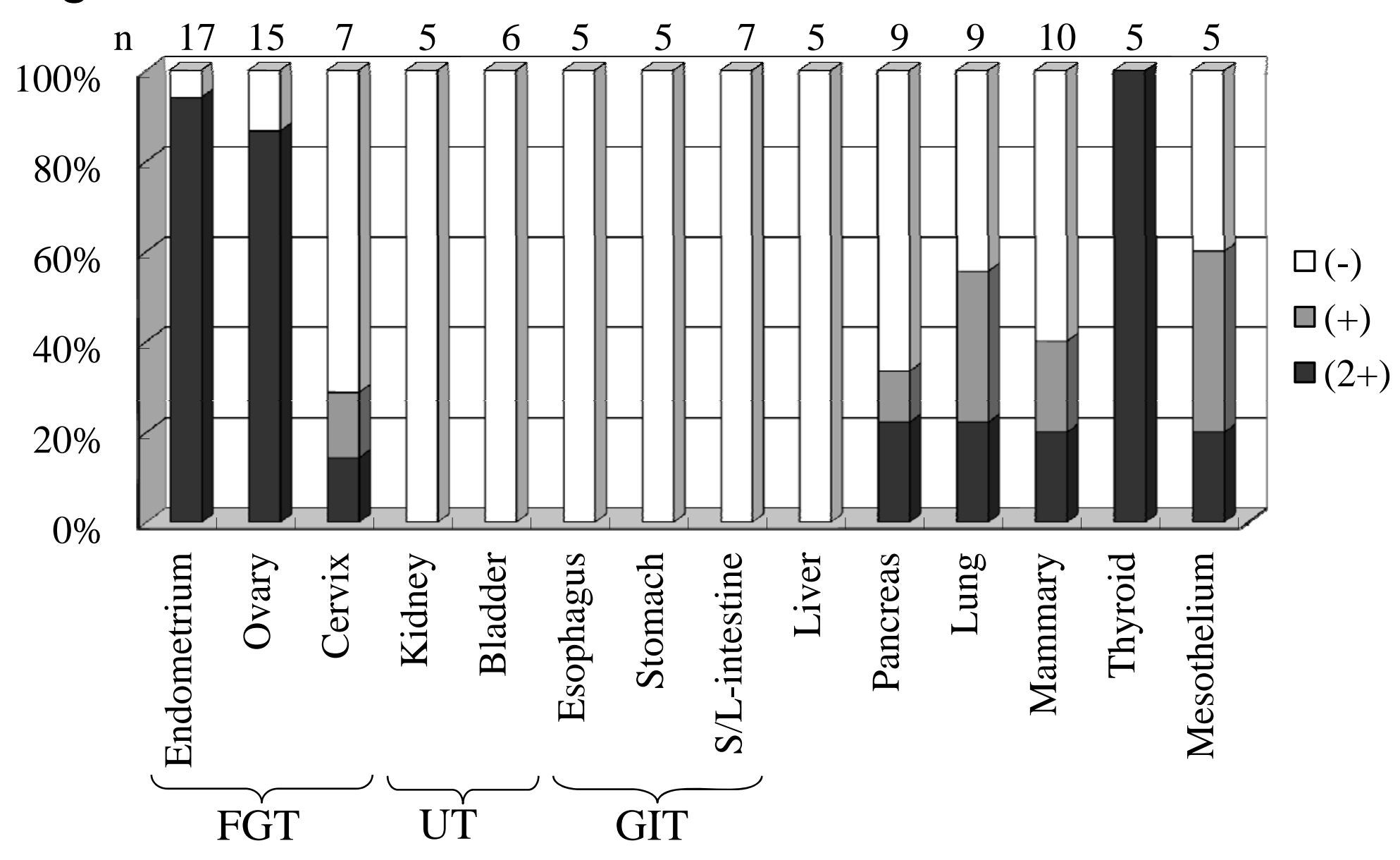


Figure 4
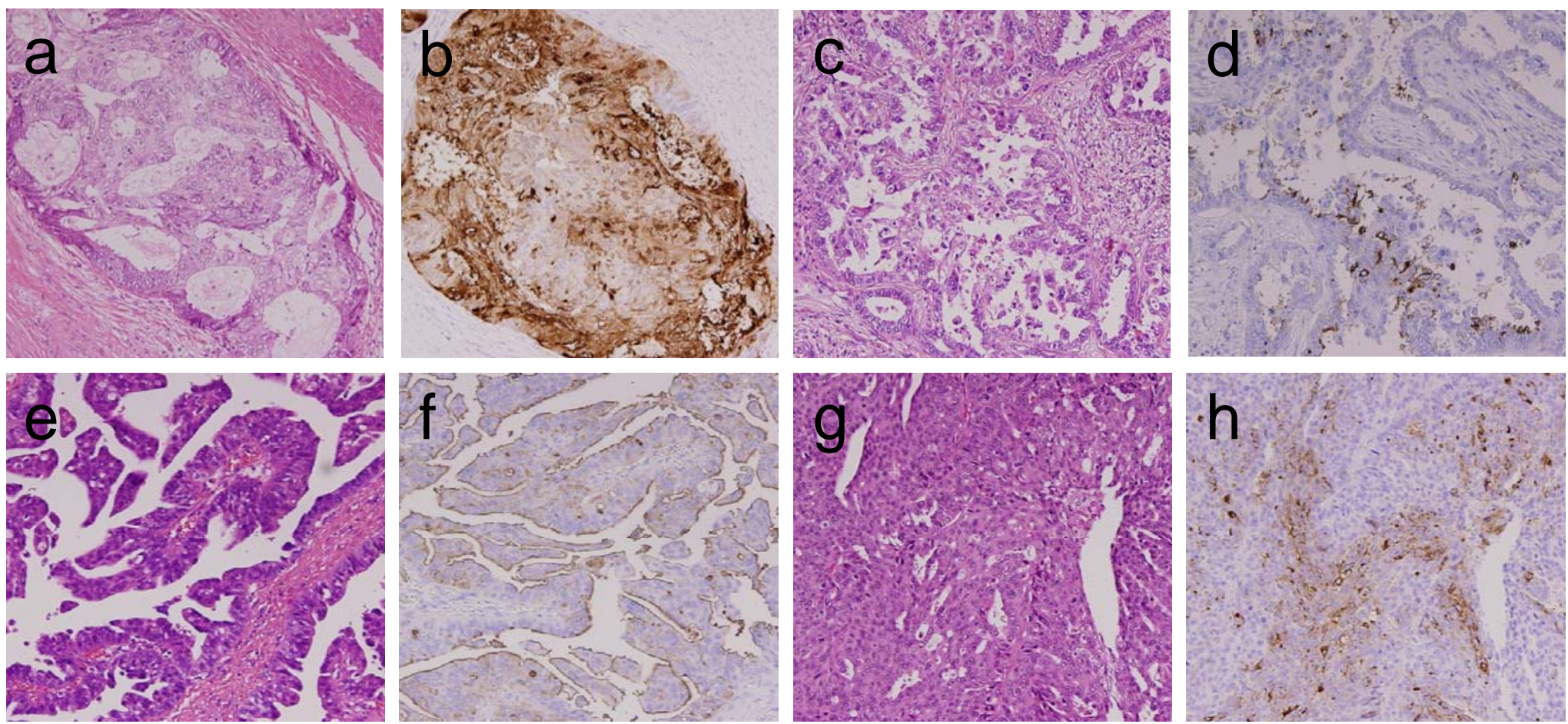


\section{Figure 5}
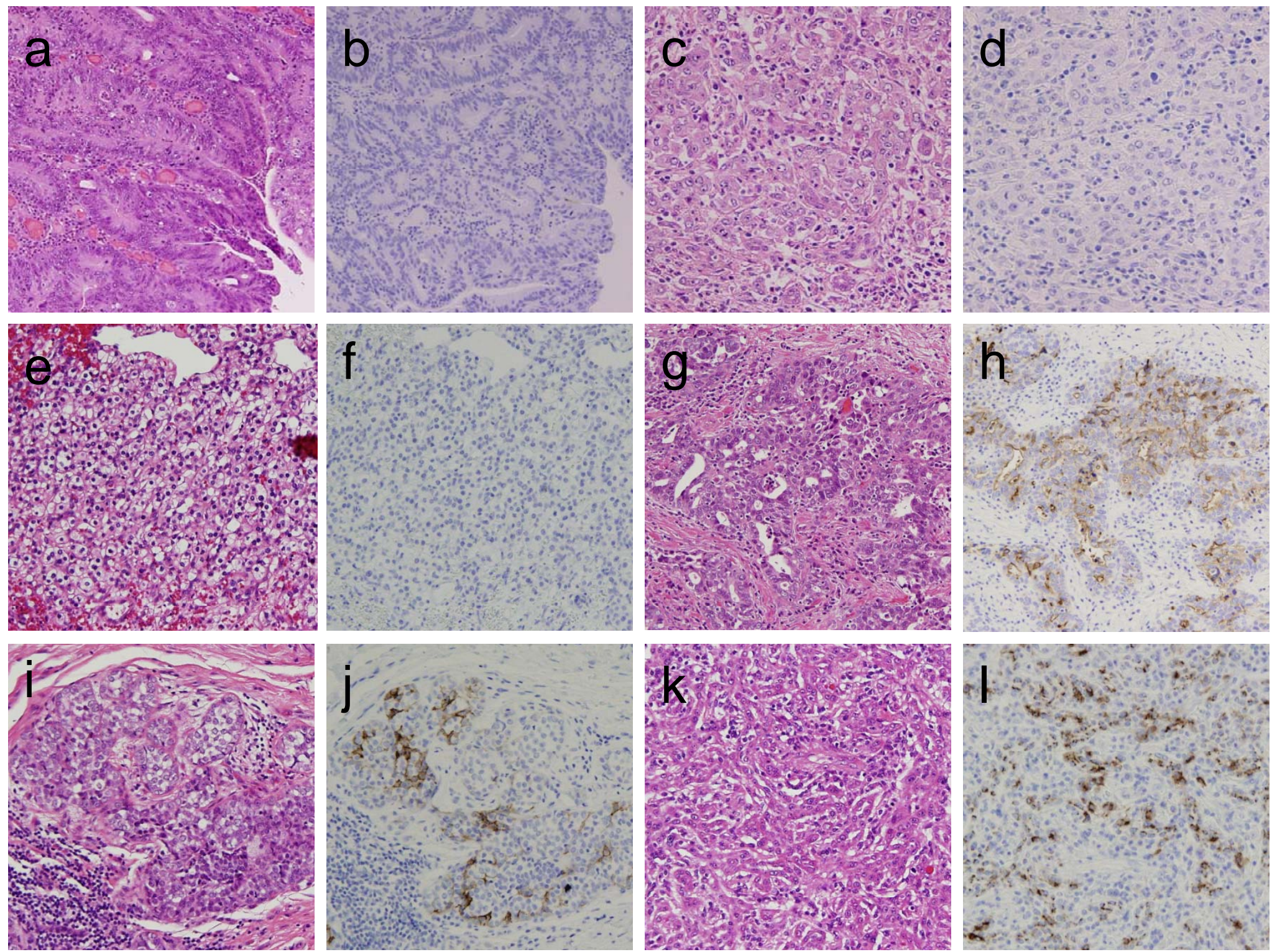